\section{Viscogonioplasty: an effective procedure for lowering intraocular pressure in primary angle closure glaucoma}

D Varma, O Baylis, N Wride, PS Phelan and SG Fraser
Department of

Ophthalmology, Sunderland Eye Infirmary, Sunderland,

Tyne and Wear, UK

Correspondence: SG Fraser, Department of

Ophthalmology, Sunderland Eye Infirmary, Queen Alexandra Road, Sunderland, Tyne and Wear SR2 9HP, UK

Tel: +44 191569 9853;

Fax: + 441915699060 .

E-mail: sfraser100@

totalise.co.uk

Received: 10 August 2005 Accepted in revised form: 28 October 2005

Published online: 13 January 2006

\begin{abstract}
Purpose To evaluate the safety and intraocular pressure (IOP) lowering effect of combined phacoemulsification and viscogonioplasty (Phaco-VGP) in managing primary acute closed-angle glaucoma (ACAG) unresponsive to conventional therapy (patent PI).

Patient and Methods In all, 15 consecutive eyes of patients with refractory ACAG and greater than $270^{\circ}$ peripheral anterior synechiae (PAS) underwent VGP. The technique of VGP involved routine phacoemulsification with intraocular lens implantation (Phaco/IOL) under topical anaesthetic. Following IOL implantation a heavy viscoelastic was used to deepen the anterior chamber and then injected near the angle for $360^{\circ}$ (without touching the trabecular meshwork) to break the PAS. No surgical instrument was used to physically break the PAS. Upon completion of VGP, automated irrigation with balanced salt solution to remove the viscoelastic was performed.
\end{abstract}

Results Mean IOP reduced from 52.1 to 14.1 $\mathrm{mmHg}$ by Phaco-VGP at 6-months review. 14/15 patients were free of glaucoma medications at 6-month review. All angles showed exposure of the trabecular meshwork over $360^{\circ}$ postoperatively without evidence of residual synechiae. No untoward complications were observed in any patient. Conclusion VGP may have a role in controlling IOP effectively and safely in patients with refractory ACAG. It produces a large drop in the IOP and opening of the angle. It is a relatively simple technique to learn and we would recommend its use in the eyes of all patients who have had ACAG and are undergoing cataract extraction.

Eye (2007) 21, 472-475. doi:10.1038/sj.eye.6702224; published online 13 January 2006

Keywords: acute glaucoma; angle surgery; viscogonioplasty; cataract

Introduction

Acute closed-angle glaucoma (ACAG) is the apposition or adhesion of the iris to the trabecular meshwork (TM) resulting in a decrease in aqueous outflow and increase in intraocular pressure (IOP) to clinically significant levels. Management of an ACAG attack is usually done with medical therapy and Nd:YAG laser peripheral iridotomy (PI) to the affected and fellow eye. In some cases, permanent peripheral anterior synechiae (PAS) develop and, when greater than $180^{\circ}$ of the angle is closed, the IOP rises despite medical therapy or patent PI. ${ }^{1}$ Various glaucoma operations ${ }^{2}$ have been advocated to open the angle in such patients with refractory ACAG. However, these interventions can be associated with serious side effects resulting in long-term visual deterioration. Other procedures described include laser trabeculoplasty and goniosynechialysis. Goniosynechialysis is a procedure designed to break PAS and restore trabecular filtration in eyes with angle closure. Conventionally this has been described using surgical instruments or by Nd:YAG laser. ${ }^{3-5}$ In this paper, we describe a new technique termed Viscogonioplasty (VGP) whereby a viscoelastic instead of a surgical instrument is used to break 
PAS and open the angle. As far as we know this is the first time that this 'non touch' procedure has been described in the literature.

\section{Purpose}

To evaluate the IOP lowering effect of combined phacoemulsification and viscogonioplasty (Phaco-VGP) in managing primary ACAG unresponsive to conventional therapy (PI).

\section{Patient and methods}

In all, 15 consecutive eyes of patients with primary ACAG were included in the study. Refractory ACAG was defined as ACAG patients where IOP was raised despite medical therapy and patent laser PI. All the patients included in the case series had suffered a classic (ie no cause other than anatomic predisposition was identified) attack of ACAG.

Inclusion criteria included iris occluding the trabeculum for at least $270^{\circ}$ of the angle as confirmed by gonioscopy, uncontrolled IOP (without anti-hypertensive medication) and the presence of a patent laser PI to eliminate pupillary block. Exclusion criteria included plateau iris, other glaucomas, previous glaucoma surgery (ALT/trabeculectomy), retinal disease and history of ocular injury. The duration of increase IOP or synaechiel angle closure was not one of our inclusion or exclusion criteria but the maximum length of time since the acute attack was no more than 4 months.

Preoperatively, all patients underwent complete ocular examination including visual acuity (VA) by Snellen' testing, IOP measurement, slit-lamp biomicroscopic examination of anterior and posterior segment. The same glaucoma surgeon performed phaco-VGP in all the cases. The operations were performed between April 2002 and December 2004. Postoperative therapy included oral acetazolamide $(250 \mathrm{mg}$ ) four times a day for $24 \mathrm{~h}$, topical steroid (1\% prednisolone acetate) four times a day and topical ketorolac drops, four times a day in the operated eye. Other antiglaucoma medications were discontinued and restarted according to IOP response. To evaluate the medical antiglaucomatous treatment, we used a scoring system as used by Kubota et al. ${ }^{6}$ In this one point was assigned for each antiglaucomatous medication and two points for oral acetazolamide. At each postoperative follow-up, complete ocular examination including gonioscopy was done in all the patients.

\section{Technique of viscogonioplasty}

Patients underwent routine phacoemulsification with intraocular lens (IOL) implantation under topical anaesthetic. Phacoemulsification was performed via a $3.2 \mathrm{~mm}$ temporal incision as a standard method and a $5.5 \mathrm{~mm}$ foldable acrylic posterior chamber IOL was implanted in the bag in all the cases. Following IOL implantation a heavy viscoelastic (Artivisc plus ${ }^{\circledR}$ ) was used to deepen the anterior chamber and then injected near the angle for $360^{\circ}$ (without touching the TM) to break the PAS, taking care to avoid ciliary body recession and inadvertent injury to the surrounding tissues. Care should also be exercised while injecting the heavy viscoelastic to avoid inadvertent release of the cannula and damage to angle. No surgical instrument was used to physically break the PAS. Upon completion of VGP, automated irrigation with balanced salt solution (BSS) to remove the viscoelastic was performed.

\section{Results}

The results are presented in Tables 1 and 2. In all, 15 Caucasian patients who presented to the eye department with ACAG and treated with laser PI fulfilled the study criteria. The mean age of patients was 64 years. All patients in this series had ACAG with at least $270^{\circ}$ PAS occluding the TM on gonioscopy. The mean IOP without any anti-glaucoma treatment (ie the presenting IOP) was $52.1 \mathrm{mmHg}$ while immediate preoperative mean IOP (on anti-glaucoma medication) was $27.4 \mathrm{mmHg}$. All patients were on at least two medications (range 2-4). Eight patients were on oral acetazolamide. Status of lens in the patients included from early to moderate lens opacity. The patients were followed-up for at least 6 months. Mean follow-up of patients was 10.6 months, (range 6-24 months).

Postoperative state of patients was as follows:

\section{Intraocular pressure}

Following combined cataract extraction and VGP mean immediate preoperative IOP reduced from $27.4 \mathrm{mmHg}$ on anti-glaucoma therapy to $14.1 \mathrm{mmHg}$ without any treatment at 6 months review in majority (14/15) of the study cases. Out of 15 , six (40\%) eyes completed 1-year follow-up and mean IOP at 1 year was $13.6 \mathrm{mmHg}$. None of the patients needed subsequent trabeculectomy or any other surgical intervention to lower IOP. Out of 15 eyes, in 14 eyes (93\%) the IOP was controlled without any antiglaucoma therapy. Post operatively none of the patients needed oral acetazolamide.

\section{Gonioscopy}

Postoperatively gonioscopy showed opening of angle in all the patients. All study patients had the previously occluded TM exposed over $360^{\circ}$ and to date there have 
Table 1 Patient data

\begin{tabular}{|c|c|c|c|c|c|c|c|c|}
\hline \multirow[t]{2}{*}{ Case no. } & \multirow[t]{2}{*}{ Sex } & \multirow[t]{2}{*}{ Age (years) } & \multirow[t]{2}{*}{ Laterality } & \multicolumn{2}{|c|}{$\begin{array}{l}\text { Preoperative } \\
\text { IOP }(m m H g)\end{array}$} & \multirow[t]{2}{*}{$\begin{array}{l}\text { Preoperative } \\
\text { medication }\end{array}$} & \multirow[t]{2}{*}{$\begin{array}{l}\text { Postoperative IOP } \\
m m H g \text { ( } 6 \text { months) }\end{array}$} & \multirow[t]{2}{*}{$\begin{array}{l}\text { Postoperative } \\
\text { medication }\end{array}$} \\
\hline & & & & Without treatment & With treatment & & & \\
\hline 1 & $\mathrm{~F}$ & 76 & $\mathrm{~L}$ & 47 & 32 & $4^{\mathrm{a}}$ & 12 & 0 \\
\hline 2 & $\mathrm{M}$ & 42 & $\mathrm{R}$ & 46 & 27 & $4^{\mathrm{a}}$ & 12 & 0 \\
\hline 3 & $\mathrm{~F}$ & 70 & $\mathrm{R}$ & 44 & 36 & 2 & 18 & 0 \\
\hline 4 & $\mathrm{~F}$ & 63 & $\mathrm{~L}$ & 70 & 56 & 2 & 17 & 1 \\
\hline 5 & $\mathrm{~F}$ & 64 & $\mathrm{R}$ & 49 & 26 & 2 & 14 & 0 \\
\hline 6 & $\mathrm{~F}$ & 59 & $\mathrm{R}$ & 63 & 15 & 2 & 14 & 0 \\
\hline 7 & $\mathrm{~F}$ & 78 & $\mathrm{~L}$ & 64 & 10 & $4^{\mathrm{a}}$ & 8 & 0 \\
\hline 8 & $\mathrm{M}$ & 63 & $\mathrm{R}$ & 44 & 17 & $4^{\mathrm{a}}$ & 12 & 0 \\
\hline 9 & $\mathrm{M}$ & 51 & $\mathrm{~L}$ & 54 & 33 & 2 & 17 & 0 \\
\hline 10 & $\mathrm{M}$ & 58 & $\mathrm{R}$ & 68 & 30 & $3^{\mathrm{a}}$ & 12 & 0 \\
\hline 11 & M & 55 & $\mathrm{R}$ & 43 & 30 & 2 & 21 & 0 \\
\hline 12 & $\mathrm{~F}$ & 59 & $\mathrm{~L}$ & 54 & 22 & $3^{\mathrm{a}}$ & 10 & 0 \\
\hline 13 & $\mathrm{~F}$ & 63 & $\mathrm{R}$ & 40 & 18 & 2 & 19 & 0 \\
\hline 14 & $\mathrm{~F}$ & 63 & $\mathrm{~L}$ & 55 & 30 & $2^{\mathrm{a}}$ & 16 & 0 \\
\hline 15 & $\mathrm{~F}$ & 65 & $\mathrm{R}$ & 40 & 29 & $3^{\mathrm{a}}$ & 17 & 0 \\
\hline
\end{tabular}

${ }^{a}$ ACAG pts on oral acetazolamide.

Table 2 Pre- and postoperative IOP (mmHg) after Phaco/ $\mathrm{IOL}+\mathrm{VGP}$

\begin{tabular}{lrrr}
\hline & Eyes & Mean IOP & Range \\
\hline IOP at presentation without treatment & 15 & 52.1 & $18-70$ \\
Immediate preoperative on treatment & 15 & 27.4 & $10-56$ \\
2 weeks postoperatively & 15 & 14.6 & $8-28$ \\
3 months & 15 & 13.8 & $8-23$ \\
6 months & 15 & 14.1 & $10-21$ \\
1 year & 6 & 13.6 & $11-15$ \\
\hline
\end{tabular}

been no instances in which further synechiael closure has been observed. There is of course no guarantee that this will not occur in the long term. No dialysis or cleft of the ciliary body was observed in any patient.

\section{Complication}

Untoward intraoperative complications such as intraoperative haemorrhage and postoperative complications such as shallow AC, corneal oedema, retained viscoelastic, inflammation and iridodialysis were not observed in our cohort of patients. Posterior capsular rupture did not occur in any patient.

\section{Visual acuity}

Visual activity remained unchanged in eight eyes, improved by 1 Snellen line in four eyes, and by $2-4$ Snellen lines in three eyes. VA did not worsen in any patient following Phaco/IOL and VGP in this series.

\section{Discussion}

Patients with shallow anterior chamber and occludable angles are predisposed to pupillary block and resultant ACAG attack. During an ACAG attack in which pupillary block is the underlying mechanism, the aqueous humour in the posterior chamber is under greater pressure than the fluid in the anterior chamber causing peripheral iris to be pushed forward against the TM. Conventional management for ACAG attack includes medical therapy and laser PI. A PI allows pressure in the two chambers to equilibrate so that the peripheral iris is no longer bowed forward and thereby relieving pupillary block. In some cases, permanent PAS develop over TM causing widespread synaechial angle closure. If a greater portion of the angle has become closed $\left(270^{\circ}\right.$ or more, ie 9 clock hrs or more) medical and laser therapy is ineffective and surgical therapy becomes necessary. Filtering procedures such as trabeculectomy have been widely used. However, these procedures are associated with risk of postoperative complications such as shallow anterior chamber, choroidal detachment, malignant glaucoma and hypotonic maculopathy. ${ }^{2}$

For these reasons some practitioners have described an alternative approach known as Goniosynechialysis. Described techniques for goniosynechialysis include surgically separating the PAS from the TM with a surgical instrument under direct gonioscopic visualisation or with a Q-switched neodymium: YAG laser. $^{3-5}$ However, complications reported with this procedure include mild-to-severe haemorrhage from iris or trabeculum, fibrin exudation, choroidal haemorrhage, choroidal detachment, shallow anterior chamber and 
transient elevation of IOP in the immediate postoperative period.

Various workers have reported variable degrees of IOP lowering following a routine phacoemulsification (range 1-6 $\mathrm{mm} \mathrm{Hg}$ ) and following a combined phaco/IOL and surgical goniosynechialysis $(13-16 \mathrm{mmHg}$ ) in ACAG patients. ${ }^{16-8}$ Complications reported with surgical goniosynechialysis in these studies included fibrinoid aqueous, transient IOP elevation, photophobia and hyphema.

VGP when compared to surgical goniosynechialysis is a quick, relatively atraumatic procedure that does not require specific surgical instruments and can be performed at the time of routine phacoemulsification via the clear corneal incision.

In our case series IOP lowering was achieved with Phaco-VGP in all patients. No patient developed any of the complications previously reported with surgical goniosynechialysis. ${ }^{1,7}$ The authors suggest that the possible mechanism of action of VGP is stretching and breaking PAS thereby widening of the angle recess and flattening of the angle. Undoubtedly, the patients in this study had a mix of phacomorphic and synaechial closure, which can be difficult to differentiate in ACAG eyes. It is important, in future studies of VGP, to try to look at these groups separately.

Cataract extraction itself can, in many patients with narrow/ closed angles reduce the IOP and open the angle. ${ }^{4}$ However, without further angle procedures it is unusual to get such a large pressure drop as we have found and to remove angle synechiae. From our own ACAG patients prior to using this technique, we found much smaller pressure drops than with VGP and noticeably less postoperative exposure of the TM. We therefore feel VGP is additive to the IOP lowering effects of the direct angle procedures described above. To fully elucidate the additional benefits of VGP will require a randomised controlled trial and we are currently recruiting to this. We are also in the process of collecting intraoperative gonioscopy and ultrasonic measurements.
In the mean time we offer VGP as a simple and safe method of opening the angles and reducing the IOP in these potentially difficult eyes.

\section{Conclusion}

Our small consecutive case series has shown that VGP may have a role in controlling IOP effectively and safely in patients with refractory primary ACAG with $360^{\circ}$ PAS. Larger case series with longer follow-up are needed to substantiate these results.

\section{References}

1 Foster PJ, Aung T, Nolan WP, Machin D, Baasanhu J, Khaw PT et al. Defining' occludable angles in population surveys: drainage angle width, peripheral anterior synechiae, and glaucomatous optic neuropathy in east Asian people. $\mathrm{Br} J$ Ophthalmol 2004; 88: 486-490.

2 Wishhart S, Nagasubramanian S, Hitchings RA. Argon laser trabeculoplasty in narrow angle glaucoma. Eye 1987; 1: 567-576.

3 Senn P, Kopp B. Nd: Yag Laser synechiolysis in glaucoma due to iridocorneal angle synechiae. Klin Monatsbl Augenheilkd 1990; 196(4): 210-213.

4 Campbell DG, Vela A. Modern goniosynechiolysis for the treatment of synaechial angle closure glaucoma. Ophthalmology 1984; 91: 1052.

5 Shingleton BJ, Chang MA, Bellows AR, Thomas JV. Surgical goniosynechialysis for angle closure glaucoma. Ophthalmology 1990; 97: 551.

6 Hayashi K, Hayashi H, Nakao F, Hayashi F. Changes in anterior chamber angle width and depth after intraocular lens implantation in eyes with glaucoma. Ophthalmology 2000; 107(4): 698-703.

7 Kubota T, Toguri I, Onizuka N, Matsuura T. Phacoemulsification and intraocular lens implantation for angle closure glaucoma after the relief of pupillary block. Ophthalmologica 2003; 217: 325-328.

8 Hrasymowycz P, Papamatheakis D, Ahmed I, Assalian, Lesk $\mathrm{M}, \mathrm{Al}$-Zafiri $\mathrm{Y}$ et al. Phaco emulsification and goniosynechiolysis in the management of unresponsive primary angle closure glaucoma. J Glaucoma 2005; 14(3): 186-189. 